\title{
Individual's Breakthrough Against Habitual Destiny: The Drive and Identity of Students Resuming the College Entrance Examination
}

\author{
Xiao Jingqian ${ }^{1, \mathrm{a}^{*}}$ \\ ${ }^{1}$ School of Sociology and Population Studies, Renmin University of China, Haidian, Beijing, China \\ a*xiaojqruc@ruc.edu.cn
}

\begin{abstract}
The existence of students who choose to resume college entrance examination (Gaokao) gambling on a better result has long been a characteristic phenomenon in Chinese education. However, relevant educational policies generally inhibit this phenomenon, and these students also suffer from social discrimination. Based on solid fieldwork, this article finds that multiple factors have combined to shape the habitus of these students, and that they are vulnerable to difficulties in their efforts to break through their personal destiny and achieve class mobility. In such a situation, these students develop a distinctive identity. They perceive the value of higher academic qualifications, but often encounter difficulties in their studies due to limited educational resources, family and social limitations. The multiple factors that influence their identity create a cycle of "unfairness", "inferiority" and "pride", which is both their motivation and their shackle.
\end{abstract}

Keywords: Resuming the College Entrance Examination, Drive, Identity

\section{个体突围惯习命运：高考复读生的动力与身份认同}

\author{
当景芉 $1, a^{*}$
}

1 中国人民大学社会与人口学院, 海淀, 北京, 中国

a*xiaojqruc@ruc.edu.cn

\section{摘要}

庞大的高考复读群体是中国教育的特色现象。相关教育政策对复读学生总体上采取抑制措施, 社会上也存在对 复读学生的成见和歧视。本文基于田野调查发现，多方因素共同塑造了复读生的惯习，复读生突破个人命运、 实现阶层流动的努力容易陷入困境。在这样的处境下，复读学生形成了特征明显的学习动力和身份认同。他们 洞察到高学历的价值，但受制于有限的教育资源、家庭与社会的影响，在学业上往往遭遇困难。多方因素影响 使复读学生在身份认同上形成了以 “不公平感” “自卑感” “自豪感” 为关键词的循环，这样的矛盾既是他们的 动力, 也是他们的枷锁。

关键词: 高考复读，动力，身份认同

\section{1. 问题的提出}

在中国, 高考复读生是指已经参加过一轮高考, 但是由于不能如愿考上自己满意的学校或者其他原 因, 又回到校园复习准备再次参加高考的学生。中国 高考的竞争非常激烈, 高考复读成本很高。与这一具 有中国特色的教育现象形成对照的是, 中国教育部于 2008 年起即禁止了国内的公办高中招收复读学生, 使
复读生寻找学校的成本大为上升; 同时，复读生在中 国社会的传统观念中易被视作 “失败者”，还被指控 为侵占教育资源 (Fan, 1998)，复读生承受着隐形的 歧视，遭受社会制度结构的限制。尽管中国学界对高 考制度的研究已经汗牛充栋, 关于高考复读生的研究 却仍较为贫乏, 且方法和结论普遍较浅近, 高考复读 生始终是受凝视的客体, 被建构为需要帮扶的对象, 而缺乏复读生的主体视角。 
本文正是从此出发, 试图重现复读生做出复读决 定过程，以回应以下经验中的现实问题：在社会制度 结构的限制下, 高考复读生从哪些渠道获得学习动力 和社会支持? 学生利用 “复读” 实现阶层流动的这一 社会行动是否、如何受惯习的限制, 又是否能有突破, 有何种突破？高考复读生如何看待自己的往届生身 份？对这一符号的认同或不认同如何影响他们当前 与日后的生活与实践?

\section{2. 文献简述}

目前对高考复读现象的研究主要集中在四种视 角: 对高考复读现状进行人口学描述 (Zhao \& Chen, 2012)、分析作为理性人的复读生的复读选择 (Chen, 2001; Yu, 2008)、解读高考复读生的心理状态与压 力纾解途经 ( $\mathrm{Li}, 2016 ; \mathrm{Yu}, 2008 ; \mathrm{Wu} \& \mathrm{Cui}, 2019$ )、分 析高考复读现象对教育的影响。在最后一部分, 已有 研究的观点较为分裂: 部分文章对高考复读持负面态 度, 认为复读生加剧了高考竞争, 浪费了高中和大学 的资源, 干扰中学的正常教学, 复读生的心理状况也 令人担忧（Tian, 2007; Yang, 2005; Tang et al, 2004); 另一部分学者则对复读群体抱有同情, 认为高考复读 生在高考中处于弱势, 应该被给予更为平等的竞争机 会 (Fu \& Wang, 2010); 更为辩证综合的看法则认为 高考复读现象既存在合理性, 也存在局限（Wang \& Tan, 2014); 复读生和应届生的同时存在产生了 “政 策的公平悖论” ，即复读生的存在造成了对在读生的 不公平, 而政府关于高考复读的政策为了维护在读生 的公平又彰显出对复读生的不公平, 解开这一悖论需 要教育机会的再分配 (Huo, 2011)。

高考复读生一方面在激烈的高考竞争中奋力寻 求自身命运的突围, 另一方面又受到 “政策的公平悖 论” 制约; 既被指破坏教育公平, 又是教育资源短缺 和教育不平等的受害者。本文将聚焦这一矛盾, 呈现 高考复读生在高考竞争中的行动能力及其制约。

\section{3. 研究方法}

本文资料主要来自研究者于 2021 年 3 月在贵州省 遵义市一个下属县级市的一所复读学校的田野调研。该 复读学校距离市区约 10 公里, $95 \%$ 的学生来自本地农村 户口占比 $95 \%$, 初次高考没能上本科线的学生通常占据 $70-80 \%$ 。2 021 年该校招收有高考复读学生 103 人, 共有 在岗教师 11 人。在联系人的介绍下, 笔者征得学校领导 和老师的同意后进入田野, 在田野调查期间主要通过参 与观察法和半结构式访谈法收集资料, 并就访谈内容将 用于学术写作一事征得他们的同意。笔者以全面性和典 型性作为访谈对象选择的原则, 共访谈了 13 位复读学 生、5 位复读学校的任课教师、5 位之前在本市复读过的 学生, 以及 2 位对本地复读教育较为了解的民办学校负 责人。

需要注意的是, 出于篇幅限制, 本文并未采用调查 所得的全部资料, 但写作的构思完全基于研究者的田野 观察与互动。在本文中仅采用了 7 位典型的受访在读复
读学生的访谈资料, 其基本情况如表 1 所示。

表 1 本研究所采用访谈对象的基本情况

\begin{tabular}{|l|l|l|l|l|}
\hline 姓名 & 性别 & 年龄 & 首次高考年 & 选科 \\
\hline 包英 & 女 & 19 & 2020 & 理科英语 \\
\hline 柳洪 & 女 & 19 & 2020 & 文科英语 \\
\hline 温林 & 男 & 20 & 2020 & 理科英语 \\
\hline 何芬 & 女 & 19 & 2020 & 文科英语 \\
\hline 罗清 & 女 & 20 & 2020 & 理科英语 \\
\hline 张琪 & 女 & 19 & 2020 & 理科英语 \\
\hline 杨朋 & 男 & 21 & 2019 & 理科日语 \\
\hline
\end{tabular}

\section{4. 僧多樕少：复读生的开局境况}

学生在复读的开局面对的就是一个竞争压力大、 教育资源缺乏的复读生市场。复读生主要面临三方面 的困境。第一是激烈的竞争。2020 年, 贵州省高考报 名人数达到 47 万, 在这其中复读学生占据了近五分 之一, 且这一比例在 2017-2020 年之间逐渐上升。复 读生数量与所占比例的情况如图 1 所示。

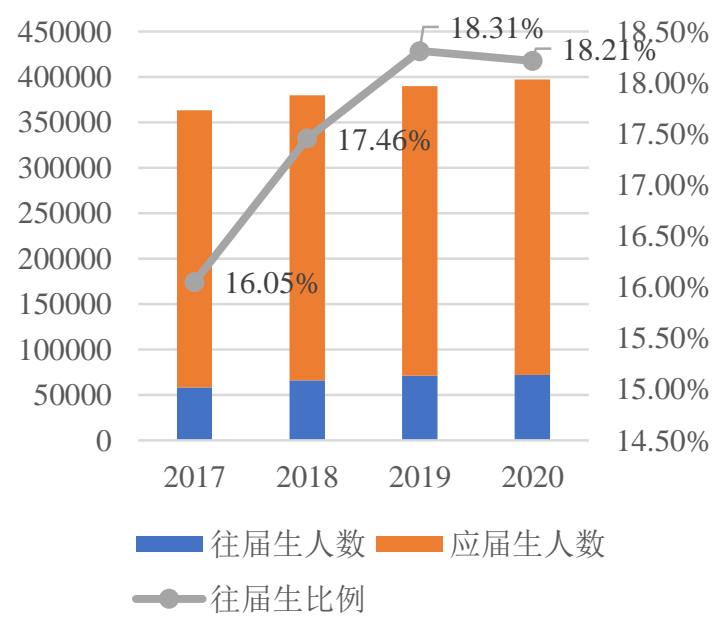

图 1 贵州省 2017-2020 年高考全国统考报名人数与 往届生比例统计图 (数据来源: 贵州省招生考试

院）

第二是公立学校过高的门槛。公立学校教学质量 普遍高于民办学校, 但只有初次高考成绩较优秀的学 生才有机会进入公立高中。在教育部的禁令落实后, 更是只有有相关人脉资源的复读生能够进入公立学 校复读。同时，公立学校的在校教师被严禁参与民办 机构的招生和教学。“禁入” 和 “禁出” 两道门槛, 同 时阻止了公立学校的教育资源外流。公办学校日渐加 高的围墙加剧了较为低分的复读学生寻找学校的困 难，成为他们面对的一大制度困境。

第三则是民办机构高价低质。但复读的学费普遍 远高于普通高中的学费, 且该数额根据上一次高考的 成绩浮动, 分越低收费越高。当地许多学生选择复读 机构, 不在意那些硬件设施、教学质量、以前的成绩, 
哪里便宜就去哪里。但同时，民办学校的设施条件和 教学质量都远不能差强人意。在研究者针对田野点学 校全体学生的问卷调查中, 只有 $15.9 \%$ 的学生对学校 的设施服务感到 “满意” , 对教学质量感到 “满意” 的学生占比仅 $28.0 \%$ 。总之, 学生在做出复读决定后, 面临的选择极为有限，他们自身突破命运的努力尝试 在一开始就进入了教育资源不平等分配的结构性枷 锁之中。

\section{5. 学生的学习动力与身份认同}

复读需要付出大量的时间成本, 还包含一定的运 气成分, 是阶层流动的惊险一跃。本文正是在这一基 础上探究复读生的动力与认同。

\section{1. 对学历价值的洞察}

高学历对复读学生有很大吸引力。杨朋是理科日 语班的高五学生, 按他的说法, 高中三年 “天天要, 没有一天是学习的, 刚来的时候连正负数都搞不懂”, 第一年高考后他大方承认, “以前不懂事, 真的不懂 事。现在改邪归正了”。此时他开始觉得 “无论如何还 是想读个本科”, “反正不管怎么说, 一定要读书”, 因 为 “但凡有点文化, 还是比那些没文化的好”。在复读 时, 他已会主动向外界寻求关于学历价值的信息, 对 于高学历的价值也越发笃信。

学生对于低学历的抵触情绪也构成复读的基本 逻辑支持。张琪高三时常被老师指派各种任务, 导致 没有时间和精力复习, 初次高考没上本科线。张琪性 格柔顺, 并不抱怨耽误了她学习的老师, 然而在原来 高中的老师劝她去省内的一所大专读幼师专业时, 她 却表现出了同尊重权威的性格截然相反的坚决态度, 觉得 “他觉得我不行, 就叫我去读幼师, 我心里面非 常抵触”, 让她去读大专是看不起她的能力, 并就在 那时做出了复读的决定。

综上, 高学历的正面刺激和低学历的负面刺激, 作为拉力和推力共同构成了学生做出复读决定的动 机。正是对学历价值洞察的程度不同, 将同一批落榜 学生中 “接受现实” 的学生和决定复读的学生区隔开 来。

\section{2. 与志向无法匹配的学习行为控制}

学生们对 “高考改变命运”普遍有较高的认同度, 但这样的洞察未必能转化为他们的行动。客观上, 学 校在对学生学习行为的规范化管理上并不严格。例如 规定 6 点起床, 但多数学生在 6:40 以后才陆续起床; 主观上, 学生们在学习方法上普遍遇到瓶颈, 对自身 的学习水平和状态没有清晰的定位与评估, 许多学生 的基础知识并不扎实, 对时间普遍缺少规划, 虽然心 急如焚, 却不知该如何突破。于是, 控制自身的学习 行为在很大程度上成了学生的自主选择与自我约束。 有些之前在公立学校就读的学生对学校管理不严多
有抱怨, “(这里) 比公立高中差很多, 那里教学水 平、管理都非常专业, 这里相对来说就比较业余” (柳 洪)。复读学校的松解管理为他们带来了困扰, 觉得 失去外部过于严肃的约束, 自己将很难集中所有精力 学习。“学习环境肯定没有那边好, 人少的话学习氛 围就不一样, 感觉没有气氛” (何芬)。学生们虽有高 考改变命运的意识和精神动力, 但难以反映到具体的 行为控制上, 这使他们的呈现出一种割裂的态度: 一 边是 “再来一次”、改变命运的孤注一掷, 另一方面则 是无法匹配的管理制度和自我控制力。学生在很大程 度上并没有发挥出潜能, 他们也难以意识到这种可能 性。

\section{3. 同时作为压力与动力的家庭}

复读生所承担的来自家庭的压力主要是由脱产 学习状态、家庭对学历的期待造成的使命感, 以及初 次高考幸负期待的愧疚感造成的。但同时, 家庭对复 读的支持也是这些学生的重要动力来源。

许多学生还是坦诚复读对家庭造成了较大经济 压力。复读生落榜后, 通常的观念会认为他/她 “本 该” 去务工赚钱, 于是当谈到生活压力时, 这种担忧 相当普遍： “你要对家庭 (负责, 作为) 一个男人你 要支撑一个家, 你不可能一直在读” (温林)。家长在 日常生活中也会不断给孩子灌输第二次高考的重要 性, 这成了学生又一重沉重的心理压力。父母离异、 来自重组家庭的包英就表示, 父亲经常向其灌输 “以 后有没有出息，就靠这一次机会了”，这给她造成很 大困扰。如此例子还有许多。

然而家庭的影响更多是正面的。绝大多数学生的 家长对于孩子复读决定都相当支持, 学生也普遍对家 庭表现出明显的亚恋, 很感激父母经济支持下的复读 机会。“我和我妈妈是小姐妹一样的。我妈妈她无条 件地支持我……但这其实也是种无形的压力, 因为她 很看好我, 不管我做什么决定她都非常支持……她是 我的动力。” (张琪) 来自单亲家庭的学生甚至可以得 到离异父母双方共同的支持。包英找到现在的复读学 校, 就是通过生身母亲的关系, 复读时现在她则与父 亲的重组家庭同住。

总之, 复读学生往往背负着更高的家庭期待, 但 来自家庭的经济和社会支持始终是复读学生学习动 力的重要来源。鼓舞和重担、感恩和逃离的矛盾交织, 家庭成了他们无法避免、必须面对的甜蜜的痛苦。

\section{4. 隐秘而迷茫的身份认同}

除了要直接面对学习的挑战和来自家庭的压力 以外, “复读生” 这一身份标签对这些学生也有相当 的影响。一方面, 多数学生仍然在意 “复读生” 的身 份。复读的学生与之前已经上大学的高中同学相处时, 总体上表现出疏远的态度。一直给研究者留下开朗自 信印象的杨朋, 谈及这个话题却多次提到复读让自己 
感觉“没面子”, “说起来都不好意思了”。出于愧疚心 态, 学生做出复读决定后, 大多会向周围的人保密。 “复读生”这一身份被他们构建出了隐蔽性。

另一方面, 复读生对公平的感知是茫然而矛盾的。 他们往往意识不到结构性的限制, 对于造成自身困境 的客观原因认知较为模糊, 在讨论相关话题时, 他们 常以茫然而模糊的态度开头, 而最终导出自己也觉得 自相矛盾的不同结论。例如, 研究者在日常聊天中曾 提到 “为什么公立学校不让招复读生” 的问题。杨朋 开始觉得 “算是公平”, 但经不住追问, 转念又觉得 “没有道理”, 力陈复读生应当有重来一次的机会。 张琪先是笃定地同意公立学校不应招复读生, 但又坦 诚复读生其实并没有占到优势, 这种优势仅仅是应届 生想象出来的。罗清则明确感受到了这种对立的矛盾, “既有同意, 也有点不太同意”, 既认为复读生 “可以 促进应届生更努力地学习”, 也指出公立学校的老师 对待复读生和应届生会有区别对待。柳洪对此愤愤不 平: “不公平, 特别不公平, 我没有选择的权利了。我 觉得应届生跟复读生就没什么区别。” 但随后又似乎 承认了复读生身份低于应届生: “可能你就没有别人 那么优秀, 即使最后结果是一样的, 但大家就觉得都 是九年义务教育，你为什么要多来一年？”

因此, 往届学生在身份认同上, 形成了 “直觉的 不公平一一自卑压抑不公平一一高考英雄的自豪一 一得到外界认同的渴望” 的循环。潜意识的自卑让他 们既认同又不认同选择面狭窄的处境, 挑战命运的自 豪感又反过来压抑这种自卑。复读生身处这种循环之 中, 往往容易陷入彷得。即使他们出于自我保护而选 择不去想这方面的问题, 呈现出茫然而矛盾的、漠不 关心的对公平的感知, 也未曾设想相关制度制定背后 的逻辑; 当需要直面这样的困境时, 他们求助于相信 自己的能动性, 认为靠复读也必然可以搏出一条出路, 高考制度的公平性和社会竞争的公平性值得信赖，而 未曾意识到自己正在以主观能动性同教育的结构性 困境作着斗争。

\section{6. 结论}

本研究通过对实地调研所获的大量资料的分析, 勾画出高考复读学生的心路历程, 分析高考复读生的 学业动力与对 “复读生” 身份的认同方式, 以及他们 的努力如何与制度限制相互拮抗。于是, 本研究初步 回答了最初提出的问题:

高考复读生的学习动力和社会支持主要来自对 阶层上升的可能性的预见与家庭、教师的支持与压力。 但是, 学生的复读实践因受制于有限的学校选择、资 源与管理水平而难以越过成绩瓶颈, 又负担着过重的 家庭期望。他们自以为有很大能动性空间, 可以靠坚 持高考改变命运，但这一能动性空间其实早就被抑制 性的复读政策所形塑为他们的惯习。惯习无法与目标 匹配, 个人意识只能带动有限的突围。高考复读生对 自身身份符号的看法则茫然而复杂, 是一个 “不公感
一自卑感一自豪感” 的循环。他们直觉地感到不公, 却出于自卑而将其过度归因于自己。然而即使如此, 复读生们却仍然乐观地相信高考制度的公平性, 认为 高考复读是坚定的决心的表现，更是使自己脱胎换骨、 心智成熟的机会。

高考制度不支持复读, 其本意是均衡分配高中教 育资源, 为职业院校引流生源, 培养技术性人才。然 而这一初衰后“只有一次机会” “只许成功不许失败” 的逻辑, 造成了社会对复读生的成见。然而, 短期内, 高考复读现象不会消失, 也不必消失。高考复读不是 “错误” 的现象, 缓解复读生困境的根本的方式, 并 不是给学生的复读之路增设种种限制, 而是减轻社会 对非本科学历的歧视和成见, 为非本科院校的学生创 造更好的就业机会, 提高这类院校的教学质量和吸引 力, 真正让青少年的发展道路呈现尽量不偏不倚的多 样性。

\section{REFERENCES}

[1] Chen, X. L. (2001) Economical Analysis of Repeating Grade 12 Phenomenon. Shanghai Jiaoyu Keyan, 10: 19-22.

[2] Fan, G R. (1998) Shilun Jiaoyu Ziyuan Langfei Jiqi dui Jiaoyu Shengtai Xitong Fazhan de Yingxiang [On the Wastage of Educational Resources And Its Impact On the Development of Educational Ecosystems]. Jiangxi Educational Research, 2: 2529.

[3] Fu, T. S., Wang, P. F. (2010) Viewing the Reform of the College Entrance Examination from the Subjective Perspective of the Students Resuming Their Studies after Graduation from Senior High School: Problems and Direction. Modern Education Management, 6: 22-25.

[4] Huo, C. F. (2011) Lun Jiaoyu Jihui de Zaifenpei Woguo Gaokao Fudu Zhengce de Gongping Beilun Jiqi Xiaojie [On the Redistribution of Educational Opportunities - The Equity Paradox of China's College Entrance Examination Restudy Policy and Its Dissolution]. Contemporary Education Sciences, 9: 7-10.

[5] Li, M. Q. (2016) Gaokao Fudu Sheng Xinli Tanxing Xianzhuang Diaocha [A Research On the Current State of Psychological Resilience of College Entrance Examination Repeaters]. Central China Normal University, Wuhan.

[6] Tang, G. F., Xu, M. (2004) Gaokao "Gaofen Fudu" Xianxiang Xianzhaung Ji Xuexi Jianyi [The Current Situation and Advice on the Phenomenon of Resuming College Entrance Examination]. Journal of Teaching and Management, 7: 77-78. 
[7] Tian, H. (2007) Analysis of the Phenomenon of Resuming University Entrance Examination in Chinese High Schools. Educational Development Research, 21: 29-33.

[8] Wu, Q. X., Cui, S. (2019) Is the Restudy Experience for the National College Entrance Examination a Barrier to Student's Career Development? Evidence from Beijing College Students Panel Survey. Education and Economy, 35(2): 57-66.

[9] Wang, K., Tan, C. F. (2014) Shilun Gaokao Fudu Xianxiang Cunzai de Helixing Jiqi Juxian [On the Reasonableness and Limits of the Existence of the Phenomenon of Resuming University Entrance Examination]. Journal of Teaching and Management, 6: $74-75,76$.

[10] Yang, P. R. (2005) Analysis of the Causes, Effects and Countermeasures of the Phenomenon of
Resuming the Gaokao with a high score. Beijing Normal University, Beijing.

[11] Yu, R. J. (2008) Social Support Network for Reattendees of College Entrance Examination-A research report based on 150 cases from Nantong, Jiangsu province, Journal of Educational Studies, 5: 82-91.

[12] Yu, X. X. (2008) Analysis of Psychological Stressor and Its Effect on Returnees Form Senior High School Graduate. Modern Preventive Medicine. 35(8): 1495-1497.

[13] Zhao, Y., Chen, W. (2012) Review on the Studies of the National College Entrance Examination Repetition from the Perspective of Demography. Social Sciences in Nanjing, 2: 84-91. 